\title{
Ánodos de Pt-Ru y Pt-Ir para Celdas de Combustible Alimentadas con Metano y Propano Directo
}

\author{
Bibian A. Hoyos ${ }^{(1) *}$, Ana I. Restrepo ${ }^{(2)}$ y Carlos M. Mesa ${ }^{(2)}$ \\ (1) Universidad Nacional de Colombia, Escuela de Procesos y Energía, Apdo. Aéreo 1027, \\ Medellín-Colombia (bahoyos@unal.edu.co) \\ (2)Universidad Nacional de Colombia, Facultad de Minas, Of. M4-214, Carrera 80 No.65-223, \\ Medellín-Colombia.
}

* autor a quien debe ser dirigida la correspondencia

\section{Resumen}

En este trabajo se evalúa el efecto de la temperatura en el desempeño de celdas de combustible de membrana de intercambio protónico alimentadas con metano y propano, utilizando oxígeno como alimentación en el cátodo. Para la oxidación de los combustibles en los ánodos, se probaron cinco catalizadores soportados en carbón: $\mathrm{Pt}, \mathrm{Pt}_{85} \mathrm{Ru}_{15}, \mathrm{Pt}_{50} \mathrm{Ru}_{50}, \mathrm{Pt}_{90} \mathrm{Ir}_{10}$ y $\mathrm{Pt}_{50} \mathrm{Ir}_{50}$. Como catalizador en el cátodo se usó platino puro soportado en carbón. El desempeño de las celdas de combustible fue evaluado mediante curvas de polarización obtenidas a partir de los datos corriente-potencial. Los resultados indican que la oxidación de metano se ve favorecida a altas temperaturas sobre los catalizadores $\mathrm{Pt}_{90} / / \mathrm{r}_{10}, \mathrm{Pt}_{50} / / \mathrm{r}_{50}$ y $\mathrm{Pt}_{50} / \mathrm{Ru}_{50}$. A bajas temperaturas los mejores catalizadores resultaron ser $\mathrm{Pt}$ y $\mathrm{Pt}_{85} / \mathrm{Ru}_{15}$. La mezcla bimetálica $\mathrm{Pt}_{85} / \mathrm{Ru}_{15}$ fue la que presentó mejor desempeño para llevar a cabo la oxidación de propano a $30^{\circ} \mathrm{C}$.

Palabras clave: celdas de combustible, ánodos de Pt-Ru y Pt-Ir, propano directo, metano directo

\section{Pt-Ru and Pt-Ir Anodes for Direct Methane and Propane Proton Exchange Membrane Fuel Cells}

\begin{abstract}
In this paper, the effect of temperature in the performance of proton exchange membrane fuel cells feed with methane and propane, using oxygen as feed to the cathode, is presented. For the fuel oxidation in the anodes, five carbon supported catalysts were tested: $\mathrm{Pt}, \mathrm{Pt}_{85} / \mathrm{Ru}_{15}, \mathrm{Pt}_{50} / \mathrm{Ru}_{50}, \mathrm{Pt}_{90} / \mathrm{Ir}_{10}$, and $\mathrm{Pt}_{50} / \mathrm{Ir}_{50}$. Carbon-supported pure platinum was used as catalysts in the cathode side. The performance of the fuel cells was evaluated by polarization curves obtained from the current-potential data. Results indicate that methane oxidation is favoured at high temperatures on the $P t_{90} / \mathrm{Ir}_{10}, \mathrm{Pt}_{50} / \mathrm{Ir}_{50}$ and $\mathrm{Pt}_{50} / \mathrm{Ru}_{50}$ catalysts. At low temperatures the best catalysts were $\mathrm{Pt}$ and $\mathrm{Pt}_{85} / \mathrm{Ru}_{15}$. The $\mathrm{Pt}_{85} / \mathrm{Ru}_{15}$ bimetallic mixture showed the best performance to carry out propane oxidation at $30^{\circ} \mathrm{C}$.
\end{abstract}

Keywords: fuel cells, Pt-Ru and Pt-Ir anodes, direct propane, direct methane 


\section{INTRODUCCIÓN}

En la actualidad, las celdas de combustible son una alternativa para la obtención eficiente de electricidad a partir de hidrocarburos de base renovable y con la cual se logra una reducción de los problemas de contaminación asociados con la combustión.

Las investigaciones más recientes en este campo se han centrado en celdas de membrana de intercambio protónico que operan con hidrógeno y metanol (Li et al., 2003; Neburchilov et al., 2007), ya que estas celdas son las que mejor se adaptan a las aplicaciones en el área automotriz (Mathias et al., 2005), para la generación de energía en áreas residenciales y en general a dispositivos móviles o estacionarios de pequeño tamaño. Aunque la eficiencia teórica de oxidación del metanol es más alta que la de otros alcoholes e hidrocarburos líquidos, su uso como combustible presenta diversos problemas: es tóxico, altamente inflamable, tiene un bajo punto de ebullición y es altamente propenso a pasar a través de la membrana de intercambio protónico (Lamy et al., 2002).

En la literatura conocida, las pruebas de desempeño para el metano y el propano en celdas de combustible han sido realmente pocas. Los escasos estudios encontrados sobre el desempeño del metano en celdas de combustible, se limitan a su uso en celdas de combustible de óxido sólido (Park et al., 1999; Zhu et al., 2006; Kronemayer et al., 2007).

Aunque el propano es un combustible con una densidad energética más alta que la del metanol, de bajo costo y fácil adquisición, son realmente pocos los estudios encontrados sobre su electrooxidación (Zhan y Barnett, 2005; Feng et al., 2007). En un trabajo que merece resaltarse (Savadogo et al., 2001) se estudió el comportamiento de una celda de combustible alimentada con propano directo utilizando $\mathrm{Pt} / \mathrm{C}, \mathrm{Pt}-\mathrm{CrO}_{3} / \mathrm{C}$ y $\mathrm{Pt}-\mathrm{Ru} / \mathrm{C}$ como catalizadores en el ánodo con una carga de platino de $2 \mathrm{mg} / \mathrm{cm}^{2}$ en cada una de las mezclas. En ese trabajo se establece que los catalizadores que exhiben mejor desempeño en cuanto a densidad de corriente son el $\mathrm{Pt}-\mathrm{CrO}_{3} / \mathrm{C}$ y el Pt-Ru/C.

El objetivo de este trabajo es evaluar el efecto de la temperatura en el desempeño de celdas de combustible de electrolito polimérico con ánodos de Pt-Ru y Pt-Ir alimentadas con metano y propano utilizando oxígeno como alimentación en el cátodo.

\section{PROCEDIMIENTO EXPERIMENTAL}

La celda de combustible empleada para este trabajo está constituida fundamentalmente por: electrodos porosos (ánodo y cátodo), electrolito polimérico (membrana de Nafion® 117), platos distribuidores de gases (de grafito conductor) y placas de cobre colectoras de electricidad. Según el diseño desarrollado por Gutiérrez et al. (2005).

Para la oxidación de los combustibles en los ánodos, se probaron cinco catalizadores soportados en carbón Vulcan® XC-72R: $\mathrm{Pt}, \mathrm{Pt}_{85} \mathrm{Ru}_{15}, \mathrm{Pt}_{50} \mathrm{Ru}_{50}, \mathrm{Pt}_{90} \mathrm{Ir}_{10}, \mathrm{Pt}_{50} \mathrm{Ir}_{50}$ con una carga de $10 \%$ en peso de platino. El cátodo se fabricó con una carga de $30 \%$ de platino puro. La cantidad del segundo metal (Ru e Ir) se adicionó según la relación deseada entre ambos metales. En la tabla 1 se listan los compuestos utilizados como fuente de los catalizadores metálicos con sus respectivas concentraciones.

Tabla 1: Fuentes de los catalizadores metálicos utilizados.

\begin{tabular}{|c|c|c|}
\hline $\begin{array}{c}\text { Nombre de } \\
\text { Compuesto }\end{array}$ & $\begin{array}{c}\text { Fórmula } \\
\text { molecular }\end{array}$ & Concentración \\
\hline $\begin{array}{c}\text { Acido } \\
\text { hexacloroplatínico } \\
\text { hexahidratado }\end{array}$ & $\mathrm{H}_{2} \mathrm{PtCl}_{6} \cdot 6 \mathrm{H}_{2} \mathrm{O}$ & $1 \mathrm{~g} \mathrm{sal} / \mathrm{ml} \mathrm{sln}$ \\
\hline Cloruro de rutenio & $\mathrm{RuCl}_{3}$. & $0.0244 \mathrm{~g} \mathrm{Ru} / \mathrm{ml} \mathrm{sln}$ \\
\hline $\begin{array}{c}\text { Hexacloroiridato de } \\
\text { sodio hexahidratado }\end{array}$ & $\mathrm{Na}_{2} \mathrm{IrCl}_{6} \cdot 6 \mathrm{H}_{2} \mathrm{O}$ & $0.0069 \mathrm{~g} \mathrm{Ir} / \mathrm{ml} \mathrm{sln}$ \\
\hline
\end{tabular}


Como agente reductor se utilizó formiato de sodio $0.5 \mathrm{M}$ con un $30 \%$ en exceso, con el fin de garantizar una completa reducción de los metales sobre el carbón. La composición de los catalizadores preparados fue verificada en un Microscopio Electrónico de Barrido (SEM- JEOL JSM 5910 LV).

Los electrodos de la celda de combustible se construyeron con una estructura conformada por una capa difusiva y una capa catalítica. Su elaboración se realizó siguiendo el procedimiento descrito por González et al. (2004). La cantidad de catalizador en cada electrodo se determinó de tal manera que en cada uno de ellos la cantidad de platino presente alcanzara los $0.5 \mathrm{mg} / \mathrm{cm}^{2}$ para los ánodos y 1.5 $\mathrm{mg} / \mathrm{cm}^{2}$ para los cátodos.

Las pruebas corriente - potencial se obtuvieron en una estación de pruebas para celdas de combustible (Electrochem Inc ${ }^{\circledR}$.). Previo a la entrada de los gases a la celda, se conectaron un par de humidificadores, los cuales fueron alimentados con agua destilada de forma permanente con el fin de garantizar las condiciones de humedad necesarias para el buen funcionamiento de la celda.

El desempeño de las celdas construidas se evaluó bajo la influencia de un único factor (la temperatura de operación de la celda) considerando tres niveles para el mismo $\left(30,50\right.$ y $\left.70{ }^{\circ} \mathrm{C}\right)$. La presión de los gases en ánodo y cátodo se mantuvo constante a lo largo de los experimentos en 10 psi.

\section{RESULTADOS Y DISCUSIÓN}

En la figura 1 se muestran los espectros obtenidos en el microscopio de electrónico de barrido para los catalizadores $\mathrm{Pt}_{85} / \mathrm{Ru}_{15}$ y $\mathrm{Pt}_{90} / / \mathrm{r}_{10}$, respectivamente. En la tabla 2 se presentan las composiciones en peso y atómicas porcentuales obtenidas a partir del mismo análisis. Los resultados obtenidos para ambos catalizadores fueron muy cercanos a los valores esperados de los cuales se partió para hacer la preparación, lo cual muestra que el método utilizado es adecuado para los fines propuestos.

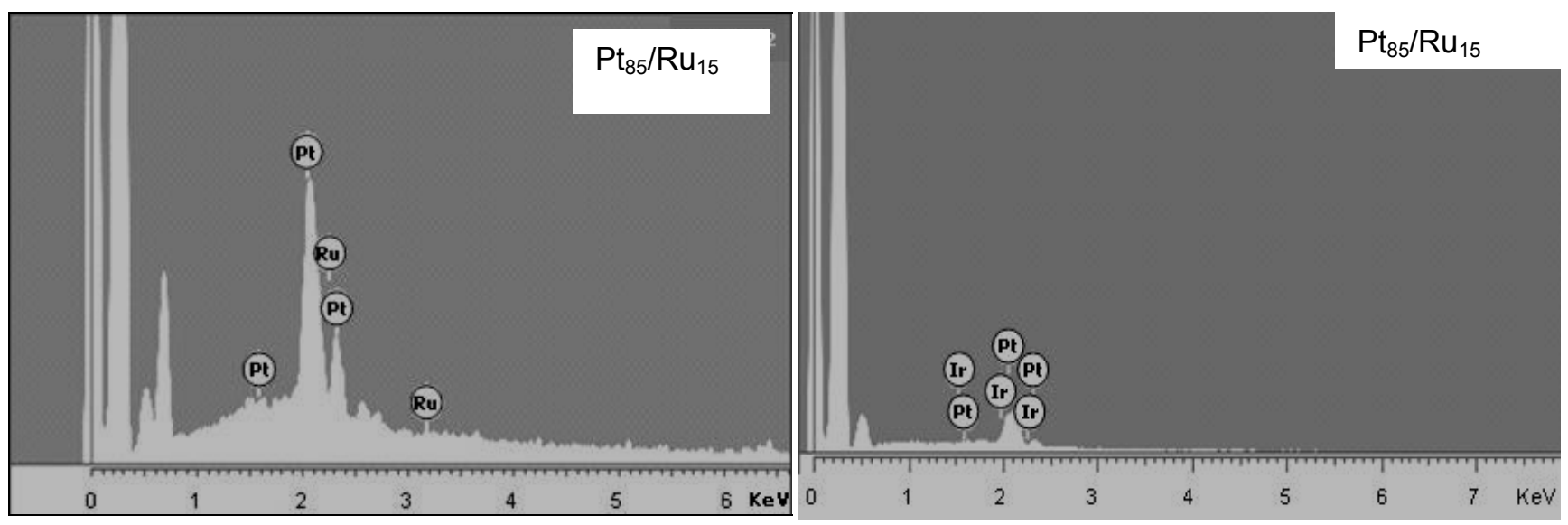

Fig. 1: Espectro EDS para los catalizadores $\mathrm{Pt}_{85} / \mathrm{Ru}_{15}$ y $\mathrm{Pt} \mathrm{t}_{90} / \mathrm{Ir}_{10}$

Tabla 2: Composiciones de las mezclas $\mathrm{Pt}_{85} / \mathrm{Ru}_{15}$ y $\mathrm{Pt}_{90} / \mathrm{Ir}_{10}$ obtenidas.

\begin{tabular}{|c|c|c|}
\hline Mezcla & \% Peso $\mathrm{Pt}$ & \% Atómico $\mathrm{Pt}$ \\
\hline $\mathrm{Pt}_{85} / \mathrm{Ru}_{15}$ & 83.59 & 72.53 \\
\hline $\mathrm{Pt}_{90} / \mathrm{rr}_{10}$ & 92.72 & 92.62 \\
\hline
\end{tabular}

\section{Operación con metano}

El desempeño de las celdas de combustible operando con metano varió notablemente con la temperatura: a $30^{\circ} \mathrm{C}$ la máxima densidad de corriente alcanzada se obtuvo con el ánodo de Pt (Fig. 2). Sin embargo, el mejor desempeño se obtuvo con $\mathrm{Pt}_{85} / \mathrm{Ru}_{15}$. A esta temperatura, la adición de 
rutenio logró mejorar la actividad catalítica del electrodo de platino. Sin embargo, a medida que la cantidad de rutenio en el electrodo se incrementa, el desempeño de la celda decae.
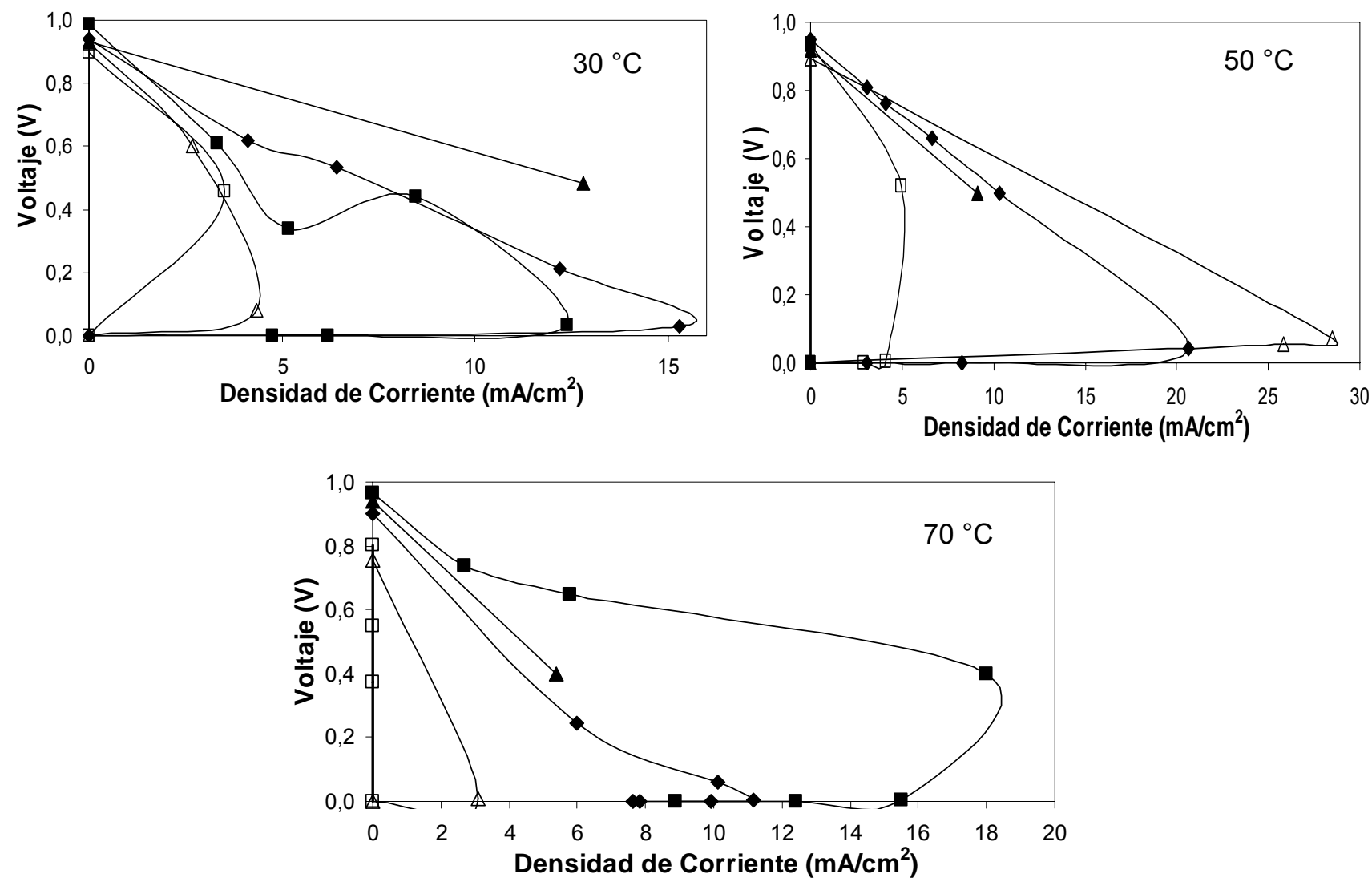

Fig. 2: Desempeño comparativo de diferentes ánodos a 30,50 y $70{ }^{\circ} \mathrm{C}$ para la oxidación de metano:

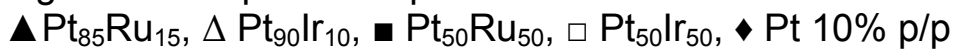

A $50{ }^{\circ} \mathrm{C}$ el ánodo de $\mathrm{Pt}_{90} / / \mathrm{r}_{10}$ superó el valor máximo de densidad de corriente obtenido a $30{ }^{\circ} \mathrm{C}$. Se puede ver que la adición de rutenio no logró aumentar el desempeño del platino, pero el incremento en la cantidad de rutenio de un 15 a un 50\% disminuyó el desempeño del par bimetálico para la oxidación de metano.

A una temperatura de $70{ }^{\circ} \mathrm{C}$, el ánodo de $\mathrm{Pt}_{50} / \mathrm{Ru}_{50}$ superó el desempeño alcanzado por los otros ánodos, sin lograr sobrepasar el valor máximo de densidad de corriente obtenido a $50{ }^{\circ} \mathrm{C}$ al usar $\mathrm{Pt} \mathrm{g}_{90} / \mathrm{Ir}_{10}$. Una mayor adición de iridio, sólo logró mejorar el desempeño obtenido con platino a $50{ }^{\circ} \mathrm{C}$.

\section{Operación con propano}

Para el propano, el ánodo de $\mathrm{Pt}_{85} / \mathrm{Ru}_{15}$ aunque no logró mantener su desempeño a altas temperaturas $\left(50\right.$ y $70^{\circ} \mathrm{C}$ ), resultó ser el mejor catalizador, sin ser superado por catalizadores como $\mathrm{Pt}$ y $\mathrm{Pt}_{50} / \mathrm{Ir}_{50}$ (Fig. 3).

A $70{ }^{\circ} \mathrm{C}$ el catalizador bimetálico $\mathrm{Pt} / \mathrm{Ru}$ resultó ser el de más bajo desempeño a todas las composiciones probadas. A 30 y $50{ }^{\circ} \mathrm{C}$ la adición de rutenio logró mejorar la actividad catalítica del electrodo de platino. Sin embargo, al aumentar la cantidad de rutenio de un 15 a un $50 \%$ el desempeño del catalizador disminuyó.

Sólo a $70{ }^{\circ} \mathrm{C}$ el par bimetálico $\mathrm{Pt}_{50} / \mathrm{Ir}_{50}$ superó el desempeño del electrodo de platino. El incremento en la cantidad de iridio resultó en un aumento en el desempeño del par bimetalico Pt/lr a altas temperaturas $\left(50\right.$ y $\left.70^{\circ} \mathrm{C}\right)$. 

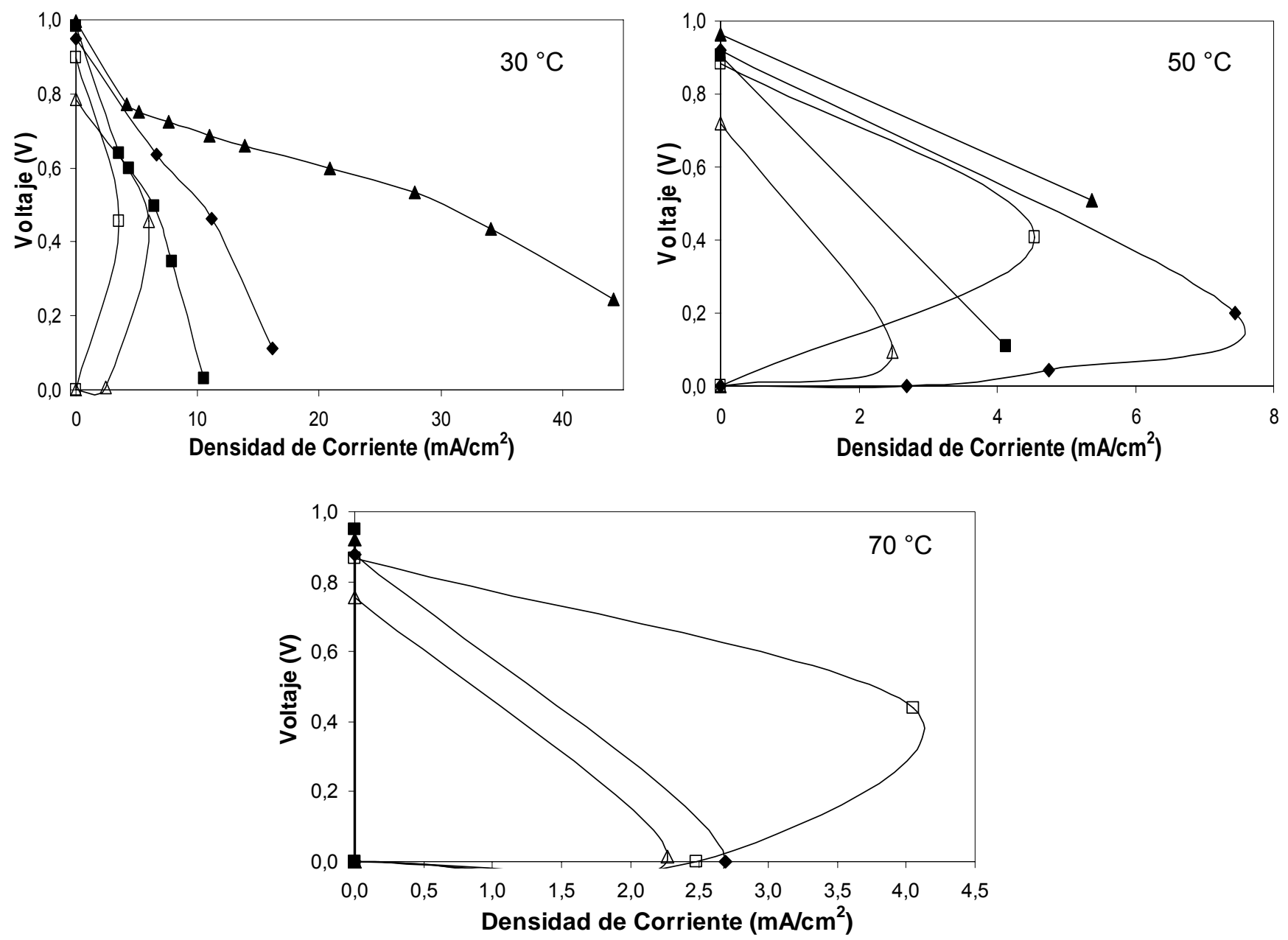

Fig. 3: Desempeño comparativo de diferentes ánodos a 30,50 y $70{ }^{\circ} \mathrm{C}$ para la oxidación de propano: $\Delta \mathrm{Pt}_{85} \mathrm{Ru}_{15}, \Delta \mathrm{Pt}_{90} \mathrm{Ir}_{10}$, - $\mathrm{Pt}_{50} \mathrm{Ru}_{50}, \square \mathrm{Pt}_{50} \mathrm{Ir}_{50}, \diamond \mathrm{Pt} 10 \% \mathrm{p} / \mathrm{p}$

En general, las corrientes obtenidas en este trabajo operando con metano y propano son muy bajas (comparadas con las que se pueden obtener con hidrogeno, por ejemplo). Aunque esto se podría atribuir a la deficiente preparación de los catalizadores, los espectros EDS muestran que las cargas y concentraciones de los metales fueron las esperadas en el momento de la preparación. La otra explicación para el bajo desempeño puede ser que la actividad a la electro-oxidación de metano y propano de las mezclas catalíticas en las condiciones de operación evaluadas en este trabajo es muy baja, lo que significa que si se desea emplear estos combustibles en una celda de combustible se deben buscar nuevos catalizadores y condiciones de operación que permitan velocidades de oxidación aceptables.

\section{CONCLUSIONES}

No todos los combustibles mostraron una tendencia clara del efecto de la temperatura sobre el desempeño de las celdas. Esto es probablemente debido a que una de las principales causas asociadas con el bajo de desempeño de las celdas de combustible es el difícil manejo de la humedad de la membrana de intercambio protónico: un exceso de agua aumenta la resistencia al transporte de masa del combustible a los sitios activos del catalizador. Pero por otro lado, una deficiencia de agua dificulta el intercambio protónico entre los electrodos de la celda.

La oxidación de metano se vio favorecida a altas temperaturas sobre los catalizadores $\mathrm{Pt} \mathrm{t}_{90} / \mathrm{Ir}_{10}$, $\mathrm{Pt}_{50} / \mathrm{Ir}_{50}$ y $\mathrm{Pt}_{50} / \mathrm{Ru}_{50}$. A bajas temperaturas los mejores catalizadores resultaron ser $\mathrm{Pt}$ y $\mathrm{Pt}_{85} / \mathrm{Ru}_{15}$.

Para la oxidación de metano a temperaturas entre 30 y $50^{\circ} \mathrm{C}$ la incorporación de un $15 \%$ de rutenio al electrodo de platino aumenta el desempeño de las celdas de combustible. Sin embargo, al pasar 
de un 15 a un $50 \%$ de rutenio la actividad catalítica para la oxidación de este combustible disminuye. A $70{ }^{\circ} \mathrm{C}$ el aumento en la cantidad del segundo metal se vio reflejado en un aumento en el desempeño de la celda.

Para la oxidación de propano, la incorporación de un 15\% de rutenio al electrodo de platino, aumenta la actividad catalítica. Sin embargo al pasar de un $15 \%$ a un $50 \%$ el desempeño de la celda disminuye.

La adición de iridio no aumenta la actividad catalítica del electrodo de platino para la oxidación de propano cuando la celda es operada a 30 y $50^{\circ} \mathrm{C}$. A $70^{\circ} \mathrm{C}$ la adición de un $50 \%$ de este metal sobre el electrodo resulta en un desempeño del mismo orden del registrado por el electrodo de $\mathrm{Pt}$.

La mezcla bimetálica $\mathrm{Pt}_{85} / \mathrm{Ru}_{15}$ fue la que presentó mejor desempeño para llevar a cabo la oxidación de propano a $30^{\circ} \mathrm{C}$.

\section{AGRADECIMIENTOS}

Este trabajo se pudo llevar a cabo gracias a la financiación de SENA-COLCIENCIAS (código del proyecto: 1118-06-12474) y de la Universidad Nacional de Colombia - sede Medellín.

\section{REFERENCIAS}

Feng, Y., J. Luo y K.T. Chuang; Conversion of propane to propylene in a proton-conducting solid oxide fuel cell, Fuel: 86 (1-2), 123-128 (2007).

González J. y otros cuatros autores; Oxidación de $\mathrm{H}_{2}$ y $\mathrm{CO}$ en una celda de combustible con ánodo de platino-estaño, Revista Ingeniería e investigación: 55, 35-40 (2004).

Gutiérrez, O. y otros seis autores; Construcción y evaluación de una celda de combustible de intercambio protónico, Dyna: 72(145), 1-11 (2005).

Kronemayer, H. y otros seis autores; Direct-flame solid oxide fuel cell (DFFC) operated on methane, propane, and butane, J. Power Sources: 166 (1), 120-126 (2007).

Lamy C. y otros cinco autores; Recent advances in the development of direct alcohol fuel cells (DAFC), J. Power Sources: 108, 283-296 (2002).

Li, Q., R. He, J.O. Jensen y N.J. Bjerrum; Approaches and Recent Development of Polymer Electrolyte Membranes for Fuel Cells Operating above $100{ }^{\circ} \mathrm{C}$. Chem. Mater: 15, 4896-4915 (2003).

Mathias, M. y otros once autores; Two fuel cell cars in every garage?, Interface: 14(3), 24-35 (2005).

Neburchilov, V., J. Martin, H. Wang y J. Zhang; A Review of Polymer Electrolyte Membranes for Direct Methanol Fuel Cells, J. Power Sources: doi:10.1016/j.jpowsour.2007.03.044 (2007)

Park, S., C. Radu, J.M. Vohs y R.J. Gorte; Direct oxidation of hydrocarbons in a solid oxide fuel cell I. Methane oxidation, J. Electrochem. Soc.:146(10), 3603-3605 (1999).

Savadogo, O. y F.L. Varela; Low-temperature direct propane polymer electrolyte membranes fuel cells (DPFC), J. New Mat. for Electrochem. Syst.: 4, 93-97 (2001).

Zhan, Z. y S.A. Barnett; Use of a catalyst layer for propane partial oxidation in solid oxide fuel cells, Solid State Ionics:176 (9-10), 871-879 (2005).

Zhu, H. y otros cuatro autores; Anode barrier layers for tubular solid-oxide fuel cells with methane fuel streams, J. Power Sources: 161 (1), 413-419 (2006). 